\title{
Updated risk models demonstrate low risk of symptomatic radionecrosis following stereotactic radiosurgery for brain metastases
}

\author{
Luke Peng ${ }^{1}$, Jimm Grimm ${ }^{1}$, Chengcheng Gui ${ }^{1}$, Colette J. Shen ${ }^{1,2}$, Kristin J. Redmond ${ }^{1}$, \\ Lindsey Sloan ${ }^{1}$, Sarah Hazell ${ }^{1}$, Joseph Moore ${ }^{1}$, Ellen Huang ${ }^{1}$, Nicholas Spoleti ${ }^{1}$, Wolfram Laub ${ }^{1}$, \\ Harry Quon ${ }^{1}$, Chetan Bettegowda ${ }^{3}$, Michael Lim³, Lawrence R. Kleinberg ${ }^{1}$
}

\footnotetext{
${ }^{1}$ Department of Radiation Oncology and Molecular Radiation Sciences, Johns Hopkins University School of Medicine, 401 N Broadway Suite 1440, Baltimore, MD, ${ }^{2}$ Department of Radiation Oncology, University of North Carolina School of Medicine, 101 Manning Dr, Chapel Hill, NC, ${ }^{3}$ Department of Neurosurgery, Johns Hopkins University School of Medicine, 600 N Wolfe Street, Phipps Building Suite 118, Baltimore, MD, USA

E-mail: Luke Peng - lpeng14@jhmi.edu; Jimm Grimm - jgrimm6@jhmi.edu; Chengcheng Gui - cgui1@jhmi.edu; Colette Shen - colette_shen@med.unc.edu; Kristin J.Redmond -kjanson3@jhmi.edu; Lindsey Sloan - lsloan2@jhmi.edu; Sarah Hazell - sarah.hazell@jhmi.edu; Joseph Moore - jamoore@jhmi.edu; Ellen Huang - ellen.huang@jhmi.edu; Nicholas Spoleti - nspolet1@jhmi.edu; Wolfram Laub -wlaub@jhu.edu; Harry Quon - hquon2@jhmi.edu; Chetan Bettegowda - cbetteg1@jhmi.edu; Michael Lim - mlim3@jhmi.edu; *Lawrence R. Kleinberg: kleinla@jhmi.edu *Corresponding author
}

Received: 30 August $18 \quad$ Accepted: 27 September $18 \quad$ Published: 15 March 19

\begin{abstract}
Background: Improvements in systemic therapy continue to increase survival for patients with brain metastases. Updated dosimetric models are required to optimize long-term safety of stereotactic radiosurgery (SRS) for this indication.

Methods: Patients at a single institution receiving SRS from December 2011 to December 2014 were retrospectively reviewed. Patients with radiographic progression of at least one lesion, and with at least 6 months of follow-up from the start of SRS were included. Grade 3 necrosis was defined as requiring surgical intervention. This data were combined with two additional published datasets to construct logistic models describing necrosis risk as a function of dose and volume.

Results: From our institution, 294 brain metastases across 57 patients in 139 treatment plans met inclusion criteria. Primary histologies included non-small cell lung cancer $(n=19)$, melanoma $(n=13)$, breast carcinoma $(n=9)$, renal cell carcinoma $(n=7)$, and other $(n=9)$. Median follow-up from SRS of first cranial metastasis was 21.7 months (range: $6.3-56.6$ ) and median overall survival was 25.6 months (range: 6.5-56.6). There were eight cases of Grade 1-2 and two cases of Grade 3 necrosis. As a useful clinical reference point, $20 \mathrm{cc}$ of total brain receiving a single-fraction equivalent dose $\geq 14$ Gy corresponded to $12.1 \%$ risk for Grade $1-3(P<0.003)$ and $3.4 \%$ risk for Grade 3 necrosis $(P<0.001)$.
\end{abstract}

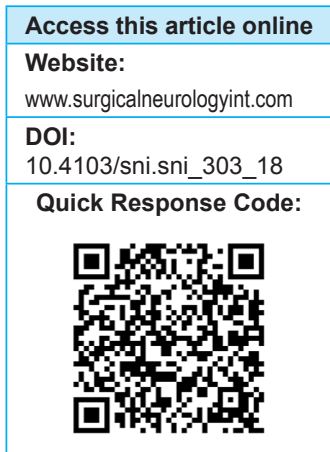

This is an open access journal, and articles are distributed under the terms of the Creative Commons Attribution-NonCommercial-ShareAlike 4.0 License, which allows others to remix, tweak, and build upon the work non-commercially, as long as appropriate credit is given and the new creations are licensed under the identical terms.

For reprints contact: reprints@medknow.com

How to cite this article: Peng L, Grimm J, Gui C, Shen CJ, Redmond KJ, Sloan L, et al. Updated risk models demonstrate low risk of symptomatic radionecrosis following stereotactic radiosurgery for brain metastases. Surg Neurol Int 2019;10:32.

http://surgicalneurologyint.com/Updated-risk-models-demonstrate-low-risk-of-symptomatic-radionecrosis-following-stereotactic-radiosurgery-for-brain-metastases/ 


\begin{abstract}
Conclusions: These results compare favorably with the QUANTEC brain tolerance estimates for radiosurgery, providing optimism for lower toxicity in the modern era. Additional studies are needed to determine dose tolerance parameters across a broad spectrum of patients.
\end{abstract}

KeyWords: Brain metastases, ionizing radiation, radiation toxicity, radionecrosis, stereotactic radiosurgery

\section{INTRODUCTION}

Stereotactic radiosurgery (SRS) has become a favored treatment option for patients with a limited number of brain metastases given its relatively low toxicity profile compared to whole brain radiation (WBRT). Though fairly uncommon, the most serious long-term complication of SRS is radiation necrosis. While headaches or neurologic deficits associated with treatment-related edema tend to be transient or tolerable with steroids, radionecrosis has the potential to induce longer lasting, progressive symptoms with some cases eventually requiring surgical intervention.

Some of the earliest work on modeling the risk of radionecrosis focused on complication rates after SRS for arteriovenous malformations (AVMs). ${ }^{[10,11,14]}$ Other early studies described more general risk models for necrosis after single fraction Gamma Knife or linear accelerator SRS, though these were largely theoretical in nature. ${ }^{[3,5,6]}$ Subsequently, the validity of these models has been explored in the context of long-term clinical experience that have led to new dosimetric parameters and clinical risk factors of interest. ${ }^{[1,4,7,8,12]}$

Given the growing ubiquity of SRS as a standalone treatment for multiple brain metastases as well as the increasing use of fractionated stereotactic radiosurgery (FSR) for larger lesions, there remains a substantial need for updated, unified, and comprehensive models for the risks of radionecrosis for brain metastases with the flexibility to incorporate varying fractionation schemes. Here, we present updated models for predicting radionecrosis risk based upon recently published datasets as well as a large institutional cohort of patients treated for brain metastases in the modern era.

\section{MATERIALS AND METHODS}

Patients who completed the CyberKnife (Accuray, Inc., Palo Alto, CA) radiosurgery from December 2011 to December 2014 for at least one intracranial metastasis in 1-5 fractions at a single institution with at least 6 months of follow-up from start of treatment were reviewed, after approval of the institutional review board. Among this set of patients, we further selected patients most likely to develop symptomatic radionecrosis by including only those with radiographic progression of at least one lesion on magnetic resonance imaging (MRI).
Cases of necrosis were defined by growth of a previously treated lesion on MRI with strong radiographic features of necrosis and/or pathologic confirmation of radiation necrosis. Grade 1 edema or radionecrosis was defined as being asymptomatic with evidence of necrosis found on imaging alone, Grade 2 was defined as being symptomatic and requiring non-surgical interventions, and Grade 3 necrosis was defined as requiring surgical intervention. In all surgical cases, pathologic confirmation of necrosis was obtained. Grade 4 necrosis was defined as life threatening and Grade 5 as death. Of note, target volumes were not subtracted from this whole brain region of interest (ROI).

These data were combined with the Inoue et al. datasets $^{[7,8]}$ which expressly reported brain dose/ volume and outcomes per patient in accordance with the recommendations of the QUANTEC ${ }^{[2,9]}$ in order that data from multiple institutions could be pooled to construct more robust models, as in the current investigation. In those studies normal brain was reported ${ }^{[7,8]}$ with gross tumor volume (GTV) subtracted, so for compatibility the median GTV volume was added back to those data prior to modeling. The Inoue et al. datasets ${ }^{[7,8]}$ were reported in terms of the volume of brain receiving 14 Gy or higher (V14Gy) in a single-fraction equivalent dose (lfxED) using the linear-quadratic (LQ) model with $\alpha / \beta=2 \mathrm{~Gy}$, so to maintain compatibility the same BED conversion was used for all data prior to modeling.

Dose calculations for each case were derived from the MultiPlan treatment planning system and plans were evaluated using the DVH Evaluator software (DiversiLabs, LLC, Huntingdon Valley, PA) by first converting all dose volume histograms (DVHs) to single-fraction equivalent doses using the LQ model with $\alpha / \beta=2 \mathrm{~Gy}$, and then performing maximum likelihood parameter fitting of normal tissue control probability (NTCP) model parameters $\operatorname{TD}_{50}(v)$, which is the $50 \%$ tolerance dose and $\gamma_{50}$, which is slope parameter of the exponential form of the logistic dose response model.: ${ }^{[15,16]}$

$$
\operatorname{NTCP}\left(D_{v}\right)=\frac{e^{4 \gamma_{50}\left(D_{v} / \operatorname{TD} 50(v)-1\right)}}{1+e^{4 \gamma_{50}\left(D_{v} / \operatorname{TD} 50(v)-1\right)}}
$$

NTCP models were thereby constructed by statistically fitting the dose distributions for all patients with and 
without toxicity to Eq. (1). This equation can be used to model a variety of $D_{v}$ (dose received by a given volume $v$ ) or $V_{\mathrm{d}}$ (volume receiving a given dose $d$ ) metrics.

\section{RESULTS}

A total of 294 brain tumors in 139 treatment plans across 57 patients in our institution met the inclusion criteria. Primary histologies included non-small cell lung cancer $(n=19)$, melanoma $(n=13)$, breast carcinoma $(n=9)$, renal cell carcinoma $(n=7)$, and other $(n=9)$. Median follow-up from SRS for first cranial metastasis was 21.7 months (range: 6.3-56.6) and median overall survival was 25.6 months (range: 6.5-56.6). No Grade 4 or greater toxicity was observed in any of the patients. There were eight cases of Grade 1-2 and two cases of Grade 3 necrosis for a total of 10 necrosis cases. In one case of Grade 3 necrosis, a renal cell carcinoma metastasis of the left parietal lobe was treated with SRS to $20 \mathrm{~Gy}$ in 1 fraction and subsequently underwent surgery 21 months later for symptomatic progression with pathology showing pure necrosis. In the second Grade 3 case, a patient with breast cancer metastatic to the right parietal lobe underwent postoperative SRS to 21 Gy in 3 fractions to the resection bed and subsequently underwent resection for progression 3 months later with pathology showing a component of residual malignancy along with extensive necrosis in the specimen. Neither of the Grade 3 necrosis patients had a history of prior radiation to the treated area. The published datasets had 223 metastases treated with the CyberKnife in 3 or 5 fractions, of which 19 had symptomatic post-SRS edema or necrosis. Five out of the 19 symptomatic lesions required resection with specimens confirming necrosis. ${ }^{[7,8]}$

The dose response model (Model A) for all patients (with toxicity grade ranging from $1-3$ ) is shown in Figure la, $P<0.003$, Wald test. Figure lb shows the second dose response model (Model B) comprising just the patients with Grade 3 toxicity, $P<0.001$, Wald test. These models provide an estimation of the full continuum of risk as a function of dose and volume. However, as a potentially useful clinical reference point in this dataset, we found that $20 \mathrm{cc}$ of total brain receiving a single-fraction equivalent dose of $14 \mathrm{~Gy}$ or more corresponded to $3.4 \%$ risk for Grade 3 and $12.1 \%$ risk for Grade 1-3 necrosis [Table 1]. A complete listing of total brain dose, volume, fractionation, and outcome for all 139 treatment plans are provided in Supplementary Table Al.

\section{DISCUSSION}

In this work, we present a pair of comprehensive NTCP models for predicting rates of radionecrosis in a contemporary cohort of patients treated with a single dose or fractionated SRS for brain metastases. Some of the key strengths of this study include its exclusive focus on brain metastases, the allowance for multiple treatment fractions (in contrast to single fraction Gamma Knife studies), and the creation of separate models for predicting severe (Grade 3) and any (Grades 1-3) necrosis.

Most of the initial groundbreaking publications on rates of SRS complications and necrosis focused on results for AVMs. Kjellberg et al. described one of the earliest models for post-SRS complications in a cohort of 74 patients treated with the proton beam therapy for

\section{Table 1: Risk of edema or necrosis as a function of volume of total brain exceeding a single-fraction equivalent dose of $14 \mathrm{~Gy}$}

\begin{tabular}{lcc}
\hline $\begin{array}{l}\text { Total brain } \\
\text { V14Gy }\end{array}$ & $\begin{array}{c}\text { Grade 1-3 edema } \\
\text { or necrosis (\%) }\end{array}$ & $\begin{array}{c}\text { Grade } \mathbf{3} \text { necrosis requiring } \\
\text { surgery (\%) }\end{array}$ \\
\hline $5 \mathrm{cc}$ & 4.1 & 0.4 \\
$10 \mathrm{cc}$ & 6.0 & 0.8 \\
$20 \mathrm{cc}$ & 12.1 & 3.4 \\
\hline
\end{tabular}

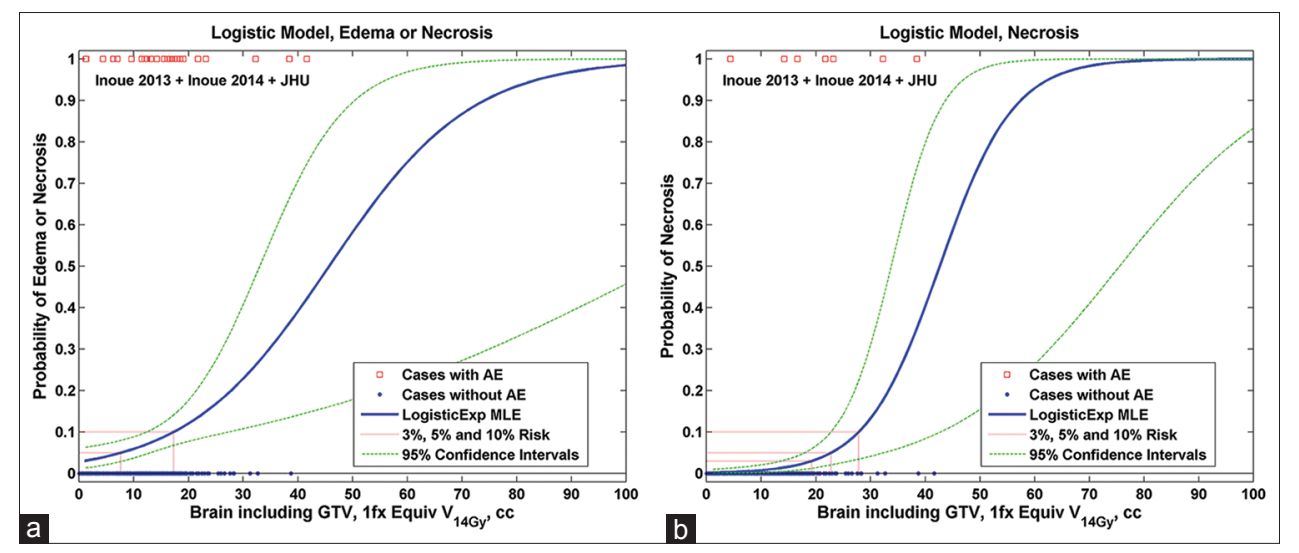

Figure I:Volume response NTCP models for brain necrosis. (a) For Grade I-3 edema or necrosis and (b) for Grade 3 surgically removed and pathologically confirmed necrosis. NTCP $=$ Normal tissue complication probability, GTV = Gross tumor volume, $c c=$ Cubic centimeters, If $=$ Single-fraction equivalent dose using linear quadratic with $\alpha / \beta=2 \mathrm{~Gy}, \mathrm{~V}$ I4Gy $=$ The volume of total brain exceeding an equivalent dose of 14 Gy in one fraction, AE = Adverse event, MLE = Maximum likelihood estimate 
AVM by plotting the $1 \%$ and $99 \%$ isoeffective dose lines for complications based on eight patients with significant treatment-related symptoms. ${ }^{[1]]}$ Subsequently, Marks and Spencer refined this work by analyzing a larger dataset of AVM patients and found that risks of symptomatic complications were substantially higher than that predicted by Kjellberg et al. $1 \%$ line. ${ }^{[14]}$ This highlights the need for continual validation and improvement of existing models to account for both accumulating clinical experience as well as shifting patterns of practice.

There was a general tendency in these early studies to conflate symptomatic complications from any cause and of any duration with actual brain necrosis. Flickinger et al. sought to more stringently define necrosis complications as "permanent" (at least 2 years) treatment-related symptoms and found that the risk of these long-term complications for AVMs treated with the Gamma Knife were predicted by the brain volume receiving 12 Gy as well as anatomic treatment location. ${ }^{[4]}$ Even this work, however, was not necessarily modeling necrosis risk directly, but rather the risk for any symptomatic complication, as evidenced by the large importance found for treatment location. While the presence of symptoms is clearly clinically relevant, accurate models predicting for actual necrosis risks require less subjective metrics. Further, models based on AVM treatment results may not be directly applicable to complication rates for brain metastases. The natural history of brain metastases is significantly shorter, the distribution of brain locations differs, and there is a greater likelihood of multiple non-adjacent metastases requiring either concurrent treatment or repeated future treatments. The common use of systemic agents like chemotherapy or immunotherapy and the possibility of additional WBRT are also potential confounders.

There have been a few major studies of necrosis rates after SRS for brain metastases. The final analysis of the Radiation Therapy Oncology Group (RTOG) 90-05 found that maximum tumor diameter as well as tumor dose were associated with Grade 3-5 neurotoxicity. ${ }^{[17]}$ Chin et al. identified a heterogeneous mix of 17 cases (including eight brain metastases) with post-Gamma Knife necrosis defined by either pathologic confirmation or necrotic appearing lesions on imaging that resolved in time. ${ }^{[1]}$ Features of necrosis cases which significantly differed from controls included glioma histology, repeat radiation treatment, treatment volume, integral dose, and 10 Gy volume (where 10 Gy was a pre-specified dose level and no other dose levels were analyzed). Korytko et al. studied a series of non-AVM lesions treated with the Gamma Knife with necrosis defined mainly by imaging and found that 12 Gy volume (again, a pre-specified dose level; other levels not investigated) correlated with symptomatic but not asymptomatic radionecrosis on logistic regression analysis. ${ }^{[12]}$ More recent studies of the CyberKnife 3-and 5-fraction treatments for brain metastases found similar associations between the volume receiving single dose equivalent 14 Gy (pre-specified dose level) and rates of radionecrosis requiring resection. ${ }^{[7,8]}$

For brain metastases treated with SRS or FSR, volumetric dose therefore appears to be a key factor for predicting rates of necrosis. The above studies, however, mostly described associations of necrosis with certain pre-specified volumetric dose levels and fell well short of creating fully comprehensive NTCP models. In terms of more complete models, Flickinger et al. early work estimated rates of complications after the Gamma Knife ${ }^{[3,6]}$ or linear accelerator $\mathrm{SRS}^{[5]}$ using the integrated logistic formula, but these models included parameters estimated from historical whole brain tolerance doses rather than actual SRS patient complications (understandable given the paucity of data available at that time). There remains a significant need for comprehensive, modern NTCP models focused on brain metastases as presented in this work.

Definitions of necrosis in many of the published datasets have been so variable and vague that the QUANTEC brain tolerance paper ${ }^{[13]}$ purposefully avoided usage of the words "grade" and "pathology," and pointed out that "biopsy is rarely performed to confirm suspected radiation necrosis." To our knowledge, Figure la and $\mathrm{b}$ are the first published data showing the effect of toxicity grading on differences in modeled brain necrosis dose/volume response. As would be expected, asymptomatic radiographic findings (Grade l) are a lot more common, so when combined with the symptomatic cases (Grade 2) and the frank necrosis that actually required surgery (Grade 3), the model in Figure la shows higher incidence at lower dose/volume with a milder slope, whereas the model of only Grade 3 in Figure la has a steeper slope and more clearly confirms the relationship of dose/volume on the outcome.

Absolute rates of necrosis after SRS were quite low in this cohort even though we only included patients from our institution who had subsequent radiographic lesion growth and therefore a higher baseline risk for developing radionecrosis. We utilized this strict inclusion criteria to provide an initial, upper bound estimate for the risks of necrosis after SRS, with the knowledge that this result is likely an overestimate of true risk. Our goal with this pilot study was to demonstrate feasibility and sensibility of results for our comprehensive modeling approach, and future work will involve broadening our inclusion criteria to all patients treated with SRS and updating the models accordingly.

There are a few possible reasons for such low toxicity rates in this patient population. In this contemporary cohort, we have had the benefit of utilizing more recently published data on necrosis risks to inform our treatment 
planning and volumetric constraints. More advanced technologies such as the CyberKnife accurate motion tracking system have likely also provided some advantages over older, frameless linear accelerator SRS systems. In our institutional practice, we tend to fractionate treatments for larger lesions or for sensitive locations to reduce complication rates while maintaining comparable rates of tumor control. We generally treat in a single fraction for lesions $<3 \mathrm{~cm}$ in size, three fractions for $3-4 \mathrm{~cm}$, and five fractions for $>4 \mathrm{~cm}$. Overall, our finding of low necrosis rates after SRS underscores the pressing need for updated models to more accurately reflect the risks faced by patients undergoing treatment for brain metastases in the modern era. These results suggest that physicians may have the option of escalating dose even further for larger lesions to improve local control while maintaining acceptably low rates of normal tissue complications.

In summary, this work describes new comprehensive NTCP models for estimating rates of necrosis after SRS for brain metastases, based on a large contemporary cohort of patients at our institution along with cohorts from recently published datasets. Overall, we show very low rates of necrosis in this selected patient cohort and our results compare favorably with the QUANTEC brain tolerance estimates for radiosurgery. Additional studies are needed to more fully determine dose tolerance parameters across a broader spectrum of patients.

\section{Financial support and sponsorship \\ Nil.}

\section{Conflicts of interest}

Dr. Grimm designed and holds intellectual property rights to the DVH Evaluator software tool which is an FDA-cleared product in commercial use. Drs. Kleinberg and Grimm have funding from the Accuray, Inc., and NovoCure, Inc. Dr. Redmond has funding from the Elekta, Inc. and Accuray, Inc.

\section{REFERENCES}

I. Chin LS, Ma L, DiBiase S. Radiation necrosis following gamma knife surgery: A case-controlled comparison of treatment parameters and long-term clinical follow up. J Neurosurg 2001;94:899-904.
2. Deasy JO, Bentzen SM, Jackson A, Ten Haken RK, Yorke ED, Constine LS, et al. Improving normal tissue complication probability models: The need to adopt a "data-pooling" culture. Int J Radiat Oncol Biol Phys 20I0;76 (3 Suppl):SI5I-4.

3. Flickinger JC. An integrated logistic formula for prediction of complications from radiosurgery. Int J Radiat Oncol Biol Phys 1989;17:879-85.

4. Flickinger JC, Kondziolka D, Lunsford LD, Kassam A, Phuong LK, Liscak R, et al. Development of a model to predict permanent symptomatic postradiosurgery injury for arteriovenous malformation patients. Arteriovenous Malformation Radiosurgery Study Group. Int J Radiat Oncol Biol Phys 2000;46: I 143-8.

5. Flickinger JC, Schell MC, Larson DA. Estimation of complications for linear accelerator radiosurgery with the integrated logistic formula. Int J Radiat Oncol Biol Phys 1990; 19:143-8.

6. Flickinger JC, Steiner L. Radiosurgery and the double logistic product formula. Radiother Oncol 1990;17:229-37.

7. Inoue HK, Sato H, Seto K, Torikai K, Suzuki Y, Saitoh J, et al. Five-fraction CyberKnife radiotherapy for large brain metastases in critical areas: Impact on the surrounding brain volumes circumscribed with a single dose equivalent of I4 Gy (VI4) to avoid radiation necrosis. J Radiat Res 2014;55:334-42.

8. Inoue HK, Seto K, Nozaki A, Torikai K, Suzuki Y, Saitoh J, et al. Three-fraction CyberKnife radiotherapy for brain metastases in critical areas: Referring to the risk evaluating radiation necrosis and the surrounding brain volumes circumscribed with a single dose equivalence of I4 Gy (VI4). J Radiat Res 2013;54:727-35.

9. Jackson A, Marks LB, Bentzen SM, Eisbruch A, Yorke ED, Ten Haken RK, et al. The lessons of QUANTEC: Recommendations for reporting and gathering data on dose-volume dependencies of treatment outcome. Int J Radiat Oncol Biol Phys 2010;76 (3 Suppl):SI55-60.

10. Kjellberg RN, Abe M. Stereotactic Bragg peak proton beam therapy. In: Lunsford LD, editor. Modern Stereotactic Neurosurgery. Boston, MA: Springer US; 1988. p. 463-70.

II. Kjellberg RN, Hanamura T, Davis KR, Lyons SL, Adams RD. Bragg-peak proton-beam therapy for arteriovenous malformations of the brain. $\mathrm{N} \mathrm{Engl}$ J Med 1983;309:269-74.

12. Korytko T, Radivoyevitch T, Colussi V, Wessels BW, Pillai K, Maciunas RJ, et al. 12 Gy gamma knife radiosurgical volume is a predictor for radiation necrosis in non-AVM intracranial tumors. Int J Radiat Oncol Biol Phys 2006;64:419-24.

13. Lawrence YR, Li XA, el Naqa I, Hahn CA, Marks LB, Merchant TE, et al. Radiation dose-volume effects in the brain. Int J Radiat Oncol Biol Phys 2010;76 (3 Suppl):S20-7.

14. Marks LB, Spencer DP. The influence of volume on the tolerance of the brain to radiosurgery. J Neurosurg 1991;75:177-80.

15. Moiseenko V, Song WY, Mell LK, Bhandare N. A comparison of dose-response characteristics of four NTCP models using outcomes of radiation-induced optic neuropathy and retinopathy. Radiat Oncol 201 I;6:6I.

16. Okunieff $P$, Morgan D, Niemierko A, Suit HD. Radiation dose-response of human tumors. Int J Radiat Oncol Biol Phys 1995;32:1227-37.

17. Shaw E, Scott C, Souhami L, Dinapoli R, Kline R, Loeffler J, et al. Single dose radiosurgical treatment of recurrent previously irradiated primary brain tumors and brain metastases: Final report of RTOG protocol 90-05. Int J Radiat Oncol Biol Phys 2000;47:291-8. 
Table A1: Total brain dose, volume, fractionation, and outcome for 139 treatment plans. $D_{v}$ values are in terms of physical dose $(\mathrm{Gy})$ and $V_{\mathrm{d}}$ values are in terms of absolute volume (cc). Example: In a single treatment session, the first patient in the table had a maximum point dose in the brain of $28.26 \mathrm{~Gy}$, with $5 \mathrm{cc}$ of brain including the GTV itself exceeding 9.58 Gy, with the median total brain dose of only $0.22 \mathrm{~Gy}$, and only $5 \%$ of the total brain exceeding a conventional fraction dose of $1.8 \mathrm{~Gy}$. The volumes exceeding $14 \mathrm{~Gy}, 12 \mathrm{~Gy}$, and $10 \mathrm{~Gy}$, were $3.05 \mathrm{cc}, 3.78 \mathrm{cc}$, and $4.76 \mathrm{cc}$, respectively. All doses have already been converted to single-fraction equivalents prior to modeling, so the patient in the second row received a maximum point dose of $1 \mathrm{fxED}=21.70 \mathrm{~Gy}$, therefore the physical dose was $36.39 \mathrm{~Gy}$ in 3 fractions, with 13.72 cc of total brain exceeding $28.8 \mathrm{~Gy}$ in 3 fractions which has a $1 \mathrm{fxED}$ of $14 \mathrm{~Gy}$

\begin{tabular}{|c|c|c|c|c|c|c|c|c|c|c|c|c|}
\hline Grade & NumFx & D50\% & D10\% & D5\% & V14Gy & V12Gy & V10Gy & D50cc & D20cc & D10cc & D5cc & Dmax \\
\hline 0 & 1 & 0.22 & 1.19 & 1.80 & 3.05 & 3.78 & 4.76 & 1.93 & 3.37 & 5.45 & 9.58 & 28.26 \\
\hline 0 & 3 & 0.71 & 2.57 & 4.08 & 13.72 & 16.82 & 20.64 & 4.57 & 10.30 & 15.75 & 16.98 & 21.70 \\
\hline 0 & 1 & 0.22 & 1.56 & 2.75 & 4.65 & 6.32 & 8.57 & 3.20 & 5.75 & 9.06 & 13.55 & 29.51 \\
\hline 0 & 1 & 0.75 & 3.64 & 6.12 & 20.27 & 25.95 & 33.25 & 7.19 & 14.10 & 18.57 & 21.46 & 26.87 \\
\hline 0 & 3 & 1.05 & 3.67 & 7.04 & 19.28 & 32.29 & 42.47 & 8.65 & 13.86 & 15.00 & 15.73 & 18.25 \\
\hline 0 & 1 & 0.41 & 2.39 & 3.53 & 6.12 & 8.62 & 12.00 & 3.97 & 7.14 & 11.12 & 15.11 & 26.87 \\
\hline 0 & 3 & 0.27 & 1.34 & 1.92 & 0.00 & 0.84 & 2.15 & 2.08 & 2.94 & 4.22 & 6.44 & 13.18 \\
\hline 0 & 1 & 1.27 & 2.80 & 3.89 & 4.89 & 7.19 & 10.48 & 4.34 & 7.02 & 10.24 & 13.89 & 25.81 \\
\hline 3 & 1 & 0.28 & 1.70 & 2.48 & 4.37 & 5.65 & 7.36 & 2.97 & 5.00 & 8.02 & 12.95 & 29.82 \\
\hline 0 & 1 & 0.37 & 1.49 & 2.18 & 3.75 & 5.15 & 6.89 & 2.69 & 4.74 & 7.69 & 12.19 & 21.67 \\
\hline 0 & 1 & 0.20 & 1.02 & 1.41 & 1.41 & 1.95 & 2.69 & 1.61 & 2.75 & 4.16 & 6.66 & 28.13 \\
\hline 0 & 5 & 0.71 & 1.91 & 2.44 & 0.91 & 1.85 & 3.24 & 2.61 & 3.77 & 5.61 & 8.25 & 19.77 \\
\hline 0 & 1 & 0.22 & 1.11 & 1.71 & 2.89 & 3.57 & 4.55 & 1.89 & 3.17 & 5.39 & 9.32 & 32.73 \\
\hline 0 & 3 & 0.61 & 3.28 & 5.42 & 8.72 & 18.09 & 27.33 & 6.60 & 11.55 & 13.73 & 14.58 & 18.51 \\
\hline 0 & 1 & 2.28 & 5.00 & 7.49 & 16.95 & 24.86 & 36.19 & 8.40 & 13.16 & 16.40 & 18.85 & 26.09 \\
\hline 0 & 1 & 0.23 & 1.64 & 2.41 & 4.36 & 5.58 & 7.13 & 2.69 & 4.48 & 7.54 & 12.89 & 25.35 \\
\hline 0 & 5 & 1.94 & 5.08 & 9.18 & 25.44 & 44.46 & 64.09 & 11.40 & 14.64 & 15.63 & 16.06 & 16.87 \\
\hline 0 & 1 & 0.74 & 2.45 & 3.41 & 6.86 & 9.07 & 12.07 & 3.57 & 6.97 & 11.30 & 16.03 & 25.00 \\
\hline 0 & 3 & 0.21 & 0.42 & 0.84 & 0.35 & 0.52 & 0.80 & 0.91 & 1.55 & 2.34 & 3.54 & 23.79 \\
\hline 0 & 1 & 1.48 & 3.90 & 5.54 & 9.41 & 12.97 & 18.02 & 5.88 & 9.43 & 13.63 & 17.70 & 30.00 \\
\hline 0 & 5 & 2.43 & 6.93 & 10.94 & 2.80 & 43.55 & 74.67 & 11.66 & 12.88 & 13.40 & 13.82 & 15.45 \\
\hline 0 & 1 & 0.22 & 1.75 & 2.45 & 2.26 & 2.97 & 4.07 & 2.87 & 4.13 & 5.89 & 8.84 & 28.98 \\
\hline 0 & 3 & 0.80 & 2.53 & 3.27 & 6.70 & 10.30 & 14.07 & 3.97 & 7.59 & 12.16 & 14.82 & 18.78 \\
\hline 0 & 1 & 0.27 & 1.27 & 1.84 & 3.09 & 3.99 & 5.26 & 2.25 & 3.87 & 6.25 & 10.36 & 32.50 \\
\hline 0 & 1 & 0.21 & 1.11 & 1.81 & 3.70 & 4.57 & 5.69 & 2.12 & 3.76 & 6.29 & 11.15 & 27.02 \\
\hline 2 & 3 & 0.88 & 3.19 & 5.56 & 19.05 & 27.59 & 35.15 & 7.13 & 13.79 & 15.04 & 15.72 & 18.18 \\
\hline 0 & 3 & 1.52 & 3.69 & 5.58 & 14.22 & 20.95 & 28.84 & 6.69 & 12.26 & 15.26 & 16.84 & 19.99 \\
\hline 0 & 1 & 0.18 & 1.15 & 1.85 & 3.12 & 4.43 & 6.13 & 2.34 & 4.21 & 7.11 & 11.29 & 23.19 \\
\hline 0 & 1 & 0.24 & 2.28 & 3.69 & 8.95 & 13.09 & 18.55 & 5.51 & 9.58 & 13.43 & 16.19 & 23.19 \\
\hline 0 & 1 & 0.44 & 3.07 & 4.62 & 0.01 & 0.01 & 0.01 & 5.56 & 9.81 & 15.09 & 19.02 & 25.81 \\
\hline 0 & 1 & 0.17 & 0.51 & 0.91 & 1.79 & 2.44 & 3.23 & 1.24 & 2.40 & 4.03 & 7.11 & 27.69 \\
\hline 0 & 1 & 0.18 & 1.12 & 1.63 & 1.22 & 1.82 & 2.63 & 1.94 & 3.00 & 4.38 & 6.77 & 25.00 \\
\hline 0 & 1 & 0.19 & 0.75 & 1.28 & 2.35 & 2.96 & 3.80 & 1.44 & 2.78 & 4.68 & 8.09 & 29.41 \\
\hline 1 & 5 & 0.45 & 1.97 & 2.57 & 0.00 & 1.00 & 5.77 & 2.79 & 4.75 & 7.62 & 10.44 & 13.20 \\
\hline 0 & 1 & 0.19 & 0.93 & 1.37 & 1.15 & 1.59 & 2.24 & 1.50 & 2.41 & 3.72 & 5.96 & 26.24 \\
\hline 0 & 3 & 0.29 & 2.18 & 2.83 & 10.81 & 13.38 & 16.48 & 3.60 & 8.26 & 14.62 & 18.56 & 22.01 \\
\hline 0 & 5 & 2.00 & 5.45 & 9.32 & 16.43 & 44.80 & 70.96 & 11.60 & 13.78 & 14.70 & 15.42 & 17.57 \\
\hline 0 & 3 & 0.62 & 2.68 & 4.53 & 22.92 & 27.84 & 33.81 & 6.85 & 15.06 & 17.62 & 18.80 & 22.01 \\
\hline 0 & 1 & 0.55 & 3.89 & 5.37 & 18.54 & 22.97 & 29.23 & 6.94 & 13.27 & 19.78 & 24.24 & 31.03 \\
\hline 0 & 1 & 0.20 & 0.59 & 1.06 & 0.87 & 1.13 & 1.50 & 1.39 & 2.05 & 2.88 & 4.39 & 34.48 \\
\hline 0 & 1 & 0.25 & 0.75 & 1.14 & 0.52 & 0.69 & 0.95 & 1.34 & 1.86 & 2.58 & 3.75 & 37.74 \\
\hline
\end{tabular}


Table A1: Contd...

\begin{tabular}{|c|c|c|c|c|c|c|c|c|c|c|c|c|}
\hline Grade & NumFx & D50\% & D10\% & D5\% & V14Gy & V12Gy & V10Gy & D50cc & D20cc & D10cc & D5cc & Dmax \\
\hline 0 & 1 & 0.11 & 0.31 & 0.49 & 0.13 & 0.26 & 0.45 & 0.60 & 1.35 & 1.87 & 2.60 & 17.24 \\
\hline 0 & 1 & 1.66 & 4.27 & 6.57 & 15.32 & 21.67 & 30.23 & 7.31 & 12.47 & 16.24 & 19.20 & 25.81 \\
\hline 0 & 1 & 0.67 & 2.38 & 3.67 & 6.83 & 10.21 & 14.40 & 4.11 & 8.05 & 12.12 & 15.34 & 23.53 \\
\hline 0 & 5 & 0.54 & 3.19 & 4.22 & 9.80 & 16.36 & 22.21 & 5.11 & 10.72 & 14.02 & 15.17 & 17.33 \\
\hline 0 & 1 & 0.31 & 1.63 & 2.36 & 5.11 & 6.64 & 8.72 & 2.93 & 5.43 & 9.05 & 14.17 & 28.13 \\
\hline 1 & 1 & 1.49 & 4.94 & 8.62 & 41.64 & 54.37 & 68.89 & 12.66 & 17.69 & 19.63 & 20.93 & 27.27 \\
\hline 0 & 1 & 0.16 & 0.34 & 0.54 & 0.64 & 0.86 & 1.17 & 0.73 & 1.23 & 2.06 & 3.44 & 27.40 \\
\hline 0 & 5 & 1.39 & 2.90 & 4.64 & 8.93 & 17.98 & 23.69 & 5.32 & 11.29 & 13.88 & 14.82 & 19.47 \\
\hline 0 & 1 & 0.19 & 0.51 & 0.71 & 0.47 & 0.61 & 0.84 & 0.85 & 1.33 & 1.96 & 2.97 & 30.77 \\
\hline 0 & 1 & 0.26 & 1.14 & 1.57 & 3.30 & 4.35 & 5.74 & 2.00 & 3.98 & 6.69 & 10.98 & 31.75 \\
\hline 0 & 3 & 1.70 & 4.39 & 7.73 & 17.95 & 32.55 & 48.48 & 9.86 & 13.70 & 14.85 & 15.48 & 19.05 \\
\hline 0 & 1 & 0.70 & 1.90 & 2.81 & 7.01 & 9.23 & 12.26 & 3.69 & 7.07 & 11.43 & 16.32 & 26.09 \\
\hline 0 & 1 & 0.73 & 2.95 & 5.24 & 12.91 & 17.99 & 25.78 & 6.76 & 11.39 & 15.50 & 19.19 & 30.28 \\
\hline 0 & 1 & 0.66 & 1.91 & 2.86 & 7.88 & 10.29 & 13.50 & 3.83 & 7.52 & 12.22 & 17.28 & 29.51 \\
\hline 0 & 1 & 0.42 & 1.81 & 2.52 & 3.36 & 4.67 & 6.43 & 2.92 & 4.69 & 7.42 & 11.60 & 23.05 \\
\hline 0 & 1 & 0.57 & 2.70 & 3.65 & 9.24 & 12.01 & 15.65 & 4.37 & 8.35 & 13.42 & 18.11 & 26.47 \\
\hline 0 & 1 & 0.18 & 0.96 & 1.62 & 2.41 & 3.37 & 4.64 & 2.08 & 3.59 & 5.74 & 9.53 & 24.00 \\
\hline 0 & 1 & 0.14 & 0.82 & 1.49 & 2.13 & 2.80 & 3.61 & 1.91 & 2.81 & 4.14 & 7.55 & 22.22 \\
\hline 0 & 1 & 0.16 & 0.66 & 1.09 & 0.74 & 1.00 & 1.36 & 1.33 & 1.90 & 2.56 & 3.93 & 25.71 \\
\hline 1 & 5 & 0.31 & 1.66 & 2.18 & 1.34 & 4.13 & 6.04 & 2.61 & 4.14 & 6.68 & 11.12 & 17.10 \\
\hline 0 & 5 & 0.42 & 1.83 & 2.60 & 0.12 & 2.81 & 9.91 & 3.21 & 6.74 & 9.98 & 11.25 & 14.66 \\
\hline 0 & 1 & 0.23 & 1.44 & 2.42 & 3.75 & 5.23 & 7.32 & 2.85 & 5.17 & 8.24 & 12.25 & 28.13 \\
\hline 0 & 5 & 0.16 & 1.01 & 1.55 & 0.00 & 0.32 & 1.38 & 1.77 & 2.49 & 3.60 & 5.47 & 12.65 \\
\hline 0 & 1 & 0.53 & 2.16 & 3.06 & 4.42 & 6.18 & 8.65 & 3.55 & 5.97 & 9.19 & 13.24 & 28.51 \\
\hline 0 & 1 & 0.79 & 2.53 & 3.70 & 9.34 & 12.41 & 16.31 & 4.44 & 8.60 & 13.53 & 17.67 & 28.13 \\
\hline 0 & 1 & 0.67 & 2.43 & 3.51 & 13.20 & 16.53 & 20.60 & 4.44 & 10.27 & 16.19 & 19.89 & 28.57 \\
\hline 0 & 1 & 0.33 & 1.10 & 1.39 & 1.29 & 1.71 & 2.27 & 1.53 & 2.31 & 3.49 & 5.57 & 29.03 \\
\hline 0 & 1 & 0.23 & 1.29 & 1.88 & 4.84 & 5.79 & 7.09 & 2.11 & 4.11 & 7.30 & 13.63 & 30.30 \\
\hline 0 & 5 & 0.74 & 3.39 & 5.70 & 9.13 & 19.18 & 30.56 & 7.46 & 11.85 & 13.89 & 14.70 & 16.03 \\
\hline 0 & 1 & 0.33 & 1.23 & 1.83 & 1.22 & 1.80 & 2.58 & 2.16 & 3.27 & 4.58 & 6.78 & 23.68 \\
\hline 0 & 3 & 0.46 & 1.64 & 2.41 & 5.28 & 7.64 & 10.55 & 3.19 & 6.34 & 10.34 & 14.20 & 19.67 \\
\hline 2 & 1 & 0.61 & 2.39 & 3.31 & 6.99 & 9.12 & 11.91 & 3.81 & 6.77 & 11.30 & 16.20 & 24.24 \\
\hline 0 & 1 & 0.31 & 1.25 & 1.71 & 1.36 & 1.97 & 2.81 & 1.95 & 3.28 & 4.77 & 7.04 & 27.12 \\
\hline 1 & 3 & 0.48 & 2.45 & 3.27 & 9.62 & 11.96 & 14.86 & 3.59 & 7.60 & 13.63 & 17.90 & 21.37 \\
\hline 0 & 1 & 0.76 & 2.42 & 3.75 & 9.59 & 12.64 & 16.51 & 4.20 & 8.64 & 13.71 & 17.71 & 23.38 \\
\hline 0 & 5 & 0.23 & 0.84 & 1.23 & 0.02 & 0.17 & 0.43 & 1.30 & 1.74 & 2.14 & 2.83 & 14.91 \\
\hline 0 & 3 & 0.88 & 3.29 & 5.26 & 18.33 & 27.91 & 36.28 & 7.47 & 13.68 & 15.03 & 15.67 & 17.29 \\
\hline 0 & 1 & 0.19 & 0.76 & 1.13 & 1.54 & 2.02 & 2.64 & 1.44 & 2.52 & 3.87 & 6.25 & 27.40 \\
\hline 0 & 1 & 0.64 & 2.30 & 3.17 & 6.25 & 8.33 & 11.19 & 3.84 & 6.76 & 10.75 & 15.45 & 27.40 \\
\hline 0 & 1 & 0.72 & 2.30 & 3.12 & 3.92 & 5.26 & 7.07 & 3.41 & 5.33 & 8.04 & 12.35 & 26.47 \\
\hline 0 & 1 & 0.57 & 2.07 & 2.79 & 2.88 & 3.88 & 5.30 & 3.04 & 4.65 & 6.83 & 10.36 & 26.47 \\
\hline 0 & 5 & 0.17 & 0.84 & 1.27 & 0.00 & 0.43 & 1.25 & 1.41 & 2.08 & 3.15 & 4.87 & 12.86 \\
\hline 0 & 1 & 0.18 & 0.50 & 0.74 & 1.13 & 1.47 & 1.92 & 0.85 & 1.67 & 2.79 & 4.72 & 29.85 \\
\hline 0 & 1 & 0.24 & 1.06 & 1.49 & 1.73 & 2.35 & 3.18 & 1.70 & 2.92 & 4.62 & 7.42 & 29.85 \\
\hline 0 & 1 & 0.22 & 1.04 & 1.50 & 1.73 & 2.38 & 3.28 & 1.73 & 3.12 & 4.91 & 7.71 & 28.57 \\
\hline 0 & 3 & 0.63 & 2.16 & 3.44 & 12.75 & 16.47 & 20.72 & 4.41 & 10.31 & 15.12 & 16.56 & 19.41 \\
\hline 0 & 1 & 0.20 & 0.66 & 1.16 & 1.37 & 1.80 & 2.41 & 1.33 & 2.08 & 3.48 & 6.05 & 33.96 \\
\hline 3 & 3 & 1.68 & 4.06 & 6.83 & 16.63 & 24.29 & 33.24 & 7.39 & 13.07 & 15.62 & 16.80 & 19.93 \\
\hline 0 & 5 & 0.26 & 2.13 & 3.31 & 10.65 & 18.13 & 23.53 & 5.00 & 11.28 & 14.19 & 15.13 & 16.87 \\
\hline
\end{tabular}


Table A1: Contd...

\begin{tabular}{|c|c|c|c|c|c|c|c|c|c|c|c|c|}
\hline Grade & NumFx & D50\% & D10\% & D5\% & V14Gy & V12Gy & V10Gy & D50cc & D20cc & D10cc & D5cc & Dmax \\
\hline 0 & 1 & 0.24 & 1.64 & 2.65 & 8.35 & 10.76 & 14.33 & 3.76 & 7.77 & 12.57 & 17.52 & 25.32 \\
\hline 0 & 5 & 1.54 & 4.20 & 8.40 & 1.16 & 11.70 & 59.47 & 10.47 & 11.57 & 12.11 & 12.64 & 16.65 \\
\hline 0 & 1 & 0.39 & 2.53 & 3.42 & 8.24 & 10.66 & 13.90 & 4.16 & 7.64 & 12.48 & 17.70 & 31.25 \\
\hline 0 & 1 & 0.31 & 2.10 & 3.15 & 7.23 & 9.53 & 12.58 & 3.91 & 7.13 & 11.62 & 16.58 & 31.25 \\
\hline 0 & 1 & 0.70 & 3.23 & 4.71 & 16.80 & 20.43 & 25.62 & 6.03 & 12.20 & 19.97 & 26.71 & 35.29 \\
\hline 0 & 5 & 2.12 & 7.59 & 12.37 & 19.64 & 77.35 & 106.3 & 13.17 & 14.06 & 14.51 & 14.89 & 16.65 \\
\hline 0 & 3 & 0.24 & 1.51 & 2.16 & 4.30 & 6.15 & 8.25 & 2.75 & 4.93 & 8.63 & 13.20 & 18.51 \\
\hline 1 & 5 & 0.79 & 2.32 & 3.56 & 1.20 & 10.31 & 16.05 & 4.54 & 8.74 & 12.10 & 13.22 & 16.03 \\
\hline 2 & 1 & 0.19 & 1.48 & 2.75 & 6.26 & 8.37 & 11.38 & 3.63 & 7.17 & 10.81 & 15.42 & 23.38 \\
\hline 0 & 1 & 1.58 & 3.32 & 4.18 & 6.50 & 8.36 & 11.16 & 4.73 & 7.15 & 10.73 & 16.23 & 34.48 \\
\hline 0 & 1 & 0.18 & 1.57 & 2.04 & 3.47 & 4.46 & 5.77 & 2.30 & 3.90 & 6.56 & 11.08 & 25.71 \\
\hline 0 & 1 & 0.18 & 0.92 & 1.38 & 2.02 & 2.61 & 3.42 & 1.67 & 3.03 & 4.67 & 7.54 & 25.64 \\
\hline 0 & 1 & 0.18 & 0.62 & 0.87 & 0.85 & 1.18 & 1.63 & 1.05 & 1.85 & 2.92 & 4.71 & 27.69 \\
\hline 0 & 1 & 0.18 & 0.84 & 1.40 & 3.24 & 4.38 & 5.97 & 1.86 & 3.94 & 7.04 & 11.11 & 24.66 \\
\hline 0 & 1 & 0.20 & 1.11 & 1.75 & 1.63 & 2.18 & 2.94 & 2.05 & 3.07 & 4.50 & 7.05 & 28.99 \\
\hline 0 & 1 & 0.31 & 1.35 & 1.97 & 3.04 & 4.03 & 5.49 & 2.44 & 4.32 & 6.81 & 10.59 & 33.90 \\
\hline 0 & 1 & 0.15 & 1.08 & 1.59 & 1.52 & 2.59 & 3.85 & 1.98 & 3.37 & 5.37 & 8.56 & 15.79 \\
\hline 0 & 1 & 0.26 & 1.21 & 1.62 & 2.08 & 2.69 & 3.58 & 1.92 & 3.29 & 5.07 & 7.99 & 34.62 \\
\hline 0 & 1 & 0.14 & 0.36 & 0.58 & 0.38 & 0.55 & 0.80 & 0.72 & 1.29 & 1.97 & 3.06 & 25.00 \\
\hline 0 & 3 & 1.61 & 4.44 & 8.31 & 38.76 & 48.59 & 60.42 & 11.74 & 17.70 & 19.26 & 19.89 & 21.96 \\
\hline 0 & 1 & 0.26 & 0.73 & 1.21 & 0.41 & 0.71 & 1.07 & 1.49 & 2.19 & 2.89 & 4.10 & 16.13 \\
\hline 0 & 1 & 0.18 & 0.53 & 0.80 & 0.45 & 0.64 & 0.89 & 0.98 & 1.60 & 2.39 & 3.52 & 28.18 \\
\hline 0 & 1 & 0.39 & 1.68 & 2.53 & 5.69 & 7.66 & 10.51 & 3.33 & 6.56 & 10.29 & 14.93 & 33.33 \\
\hline 0 & 1 & 0.71 & 2.46 & 4.02 & 13.62 & 16.60 & 20.53 & 4.65 & 10.23 & 16.90 & 21.57 & 27.69 \\
\hline 0 & 1 & 0.22 & 1.56 & 2.23 & 10.68 & 12.42 & 14.27 & 2.51 & 6.14 & 14.79 & 20.83 & 26.67 \\
\hline 0 & 1 & 0.31 & 1.56 & 2.17 & 2.82 & 3.93 & 5.44 & 2.37 & 4.14 & 6.69 & 10.52 & 25.35 \\
\hline 0 & 1 & 0.23 & 1.59 & 2.32 & 5.00 & 6.51 & 8.80 & 2.68 & 5.92 & 9.28 & 14.00 & 26.47 \\
\hline 0 & 1 & 0.42 & 1.76 & 2.45 & 1.89 & 3.14 & 4.84 & 2.71 & 4.60 & 6.79 & 9.84 & 22.22 \\
\hline 0 & 1 & 0.46 & 2.56 & 3.57 & 10.58 & 13.06 & 16.27 & 4.33 & 8.37 & 14.51 & 19.73 & 27.27 \\
\hline 0 & 5 & 0.60 & 2.58 & 3.90 & 12.34 & 18.86 & 24.88 & 5.31 & 11.61 & 14.68 & 15.75 & 17.57 \\
\hline 0 & 1 & 0.27 & 2.03 & 3.21 & 11.03 & 14.62 & 19.31 & 5.10 & 9.76 & 14.65 & 18.29 & 25.71 \\
\hline 0 & 1 & 0.55 & 1.61 & 2.60 & 3.87 & 5.29 & 7.33 & 3.15 & 5.42 & 8.26 & 12.38 & 28.57 \\
\hline 0 & 1 & 0.42 & 1.99 & 3.11 & 16.00 & 19.23 & 23.11 & 4.45 & 11.56 & 17.13 & 18.57 & 21.62 \\
\hline 0 & 5 & 2.20 & 5.10 & 8.79 & 28.31 & 43.17 & 58.25 & 11.07 & 15.13 & 16.19 & 16.88 & 19.77 \\
\hline 0 & 1 & 0.25 & 1.60 & 2.46 & 6.70 & 8.37 & 10.64 & 2.99 & 5.96 & 10.51 & 16.64 & 29.03 \\
\hline 0 & 5 & 1.04 & 2.90 & 5.00 & 0.00 & 4.55 & 35.14 & 7.24 & 10.94 & 11.50 & 11.95 & 14.07 \\
\hline 0 & 3 & 0.82 & 2.47 & 4.27 & 1.18 & 11.26 & 23.42 & 5.61 & 10.77 & 12.15 & 12.89 & 15.52 \\
\hline 0 & 1 & 0.40 & 1.56 & 2.80 & 5.28 & 7.09 & 9.66 & 3.56 & 6.25 & 9.79 & 14.38 & 31.25 \\
\hline 0 & 3 & 0.28 & 1.13 & 1.58 & 1.44 & 2.21 & 3.20 & 1.86 & 3.15 & 4.85 & 7.63 & 19.41 \\
\hline 0 & 1 & 0.49 & 1.91 & 2.87 & 8.19 & 10.16 & 12.77 & 3.51 & 6.82 & 12.15 & 18.37 & 31.75 \\
\hline 0 & 1 & 0.71 & 2.64 & 4.24 & 7.58 & 10.34 & 14.32 & 5.07 & 8.22 & 12.21 & 16.57 & 29.51 \\
\hline 0 & 3 & 1.91 & 4.60 & 8.52 & 31.24 & 38.89 & 47.98 & 9.61 & 16.69 & 18.77 & 20.04 & 23.79 \\
\hline 0 & 1 & 0.41 & 2.21 & 3.50 & 12.22 & 14.96 & 18.20 & 4.03 & 9.12 & 15.74 & 19.57 & 25.71 \\
\hline 0 & 1 & 0.25 & 2.29 & 3.36 & 10.71 & 13.15 & 16.35 & 3.83 & 8.35 & 14.67 & 19.17 & 24.24 \\
\hline 0 & 3 & 0.46 & 2.66 & 6.08 & 8.63 & 28.39 & 36.76 & 7.36 & 13.15 & 13.85 & 14.21 & 16.02 \\
\hline 0 & 1 & 0.98 & 2.98 & 4.01 & 5.29 & 7.09 & 9.90 & 4.34 & 6.84 & 9.95 & 14.39 & 27.69 \\
\hline 0 & 1 & 0.82 & 2.60 & 3.86 & 6.47 & 8.65 & 11.79 & 4.19 & 7.13 & 11.04 & 15.76 & 32.73 \\
\hline 0 & 1 & 0.60 & 1.53 & 2.14 & 3.40 & 4.55 & 6.13 & 2.35 & 4.29 & 7.09 & 11.36 & 31.03 \\
\hline 0 & 3 & 0.27 & 1.99 & 2.58 & 5.43 & 8.61 & 11.69 & 2.95 & 6.18 & 11.07 & 14.11 & 17.52 \\
\hline
\end{tabular}

